\title{
ROCKING BEHAVIOUR OF SIMPLE UNREINFORCED LOAD- BEARING MASONRY WALLS INCLUDING SOUNDPROOFING RUBBER LAYERS
}

\author{
C. Mordant ${ }^{1}$, V. Denoël ${ }^{1}$ and H. Degée ${ }^{2}$ \\ ${ }^{1}$ University of Liège \\ Chemin des Chevreuils, 1 - 4000 - Liège (Belgium) \\ e-mail: cmordant@ulg.ac.be/v.denoel@ulg.ac.be \\ ${ }^{2}$ University of Hasselt \\ Agoralaan Gebouw H - 3590 - Diepenbeek (Belgium) \\ herve.degee@uhasselt.be
}

Keywords: Unreinforced Masonry, Rocking Behaviour, Stacked Blocks, Rubber.

\begin{abstract}
This paper compares experimental measurements with predictions of different rocking models. These comparisons are carried out on the base of recent experimental results obtained by shake-table tests of four simple unreinforced load-bearing clay masonry walls that have exhibited a significant rocking behaviour for the highest acceleration inputs. In a first stage, the simple model proposed by Housner is used after slight modifications needed to properly consider the actual mass distribution. Two parameters are identified as governing the model response, namely the criterion defining the initiation of motion and the restitution coefficient. Then, a two stacked blocks model is developed and solved by an event-driven strategy. This two-block model is intended to allow a better description of the behaviour of tested specimens through a more precise modelling of the additional mass. Finally, the presence of rubber layers positioned at the top and bottom of two of the tested walls with the purpose of improving their acoustic behaviour required the development of an updated rocking model with viscous and flexible interfaces at the base of the wall and between the two stacked blocks. The properties of these interfaces are deduced from experimental data and their significant influence on the response is evidenced.
\end{abstract}




\section{INTRODUCTION}

Rocking has been originally studied by Housner in 1963 [1] with the aim of explaining the survival of tall slender structures during earthquakes. To this purpose, the equation describing the rocking motion of a rigid block resting on a rigid base has been developed under the assumption of neither sliding between the block and its foundation nor bouncing. Numerous studies and scientific contributions resulted from this original "inverted pendulum" model and the interest in this topic is still relevant today.

Some authors have used the simple model of Housner to investigate analytically and numerically the rocking response of rigid blocks subjected to a seismic action as well as their stability $[2,3,4,5]$, while others have defined a new formulation to unify the piecewise equation describing the rocking motion [6] or developed numerical tools based on the Discrete Element Method (DEM) to model the rigid block [7].

Extensions of the simple model have been carried out on various aspects. First, the assumption of a rigid support has been discussed. Psycharis and Jennings [8] introduced an elastic foundation with damping in the problem and compared two possible solutions, namely the Winkler model and a simplified unilateral two-spring model. They concluded that both solutions were equivalent, allowing the use of the simple one. The former solution was also studied in [9], whilst the latter was used in [10, 11]. ElGawady et al. [12] investigated the influence of the material constituting the interface through experimental tests and Vassilou and Makris [13] examined the benefits of different types of isolated base thanks to numerical simulations. A main outcome of this research showed that ancient classical columns of Greek temples are more stable without any isolation. Then, the consideration of sliding and bouncing has been included. Shenton III and Jones established criteria for the transition between the possible modes of response (sliding, rocking, etc.) and derived the corresponding governing equations [14]. A particular attention is given to the effects of the friction coefficients in [15]. Finally, the deformability of the block has been taken into account. Psycharis carried out a parametric analysis on a SDOF oscillator with a harmonic excitation and outlined the importance of the ratio of the natural period of the structure to the period of excitation [16]. Oliveto et al. enhanced previous models with a novel set of coordinates and transition conditions [17] and Acikgos and DeJong were focused on the interaction between elasticity and rocking [18].

All these contributions have extended the range of application defined by the simple model developed by Housner. Nevertheless, these studies were limited to a single symmetric block and horizontal bases. Plaut et al. studied the consequences of an asymmetric geometry and tilted foundations on the rocking behaviour of blocks [19]. They conclude that, in the case of asymmetric bodies, the initial direction of the excitation can significantly affect the response. Two other studies on non-symmetric rigid bodies dealt with the overturning criteria [20] and the assessment of the advantages of base isolation for such blocks [21]. The literature review has also pointed out some attempts to translate the research work into design procedures. To this purpose, Priestley et al. [22] represented the rocking block as an equivalent SDOF oscillator with damping. This analogy was however shown as unsubstantiated and oversimplified [23]. More recently, Kelly presented a design method aiming at substituting for special study required by the latest version of New Zealand code, NZS 1170.5 [24].

Besides the rocking behaviour of single block, a few researchers have been interested in the dynamic behaviour of stacked blocks although related experimental investigations are rather limited. The analytical formulation of the equations translating the rocking motion of two blocks with no sliding was developed by Psycharis [25]. Such structures can behave according to eight different configurations, as illustrated in Figure 1. Spanos et al. [26] improved 
these developments by adding different contributions to the transition criteria from one configuration to another and proposed an alternative method for the linearization of the nonlinear piecewise equations. Kounadis et al. assessed the minimum amplitude ground excitation leading to the overturning instability [27]. In order to consider these structures in design procedures, DeJong and Dimitrakopoulos proposed a methodology to derive approximate equivalence between rocking blocks and SDOF structures [28]. An experimental campaign has also been performed at the National Laboratory of Civil Engineering (LNEC) and where single blocks were tested, as well as multi-block structures lying on a rigid support [29]. This campaign allowed a better understanding of the rocking mechanisms and a comparison of measurements with model predictions.

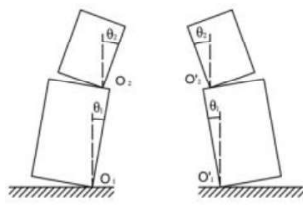

Configuration 1

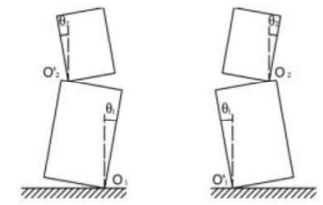

Configuration 2

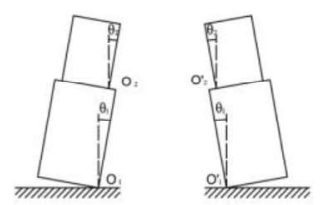

Configuration 3

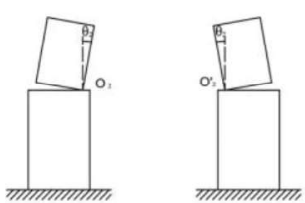

Configuration 4

Figure 1: Rocking configurations for two-block assemblies

The present contribution takes place in the general context of experimental shaking table tests carried out at the EQUALS Laboratory in the framework of the SERIES project. The tested specimens are four simple unreinforced load-bearing masonry walls. The walls have two different aspect ratios, namely 1.1 (long walls) and 0.4 (short walls), and are loaded with an additional 5-ton steel mass lying on their top. They are made of thin-bed layered clay masonry with empty vertical joints. One wall of each aspect ratio includes soundproofing rubber layers located at the wall bottom and top for acoustic reasons. Pictures of the walls are given in Figure 2. The objectives of this campaign are to enhance the understanding of the dynamic behaviour of unreinforced masonry and to investigate the influence of the rubber layers. The preliminary assessment based on equivalent static procedure predicted a shear collapse. The observed behaviour of the walls was actually characterized by a significant rocking effect, at least for the highest acceleration inputs. The importance of this rocking effect has also proved to be strongly dependent on the aspect ratio and the presence of acoustic devices. Details of this experimental campaign are given in [30].

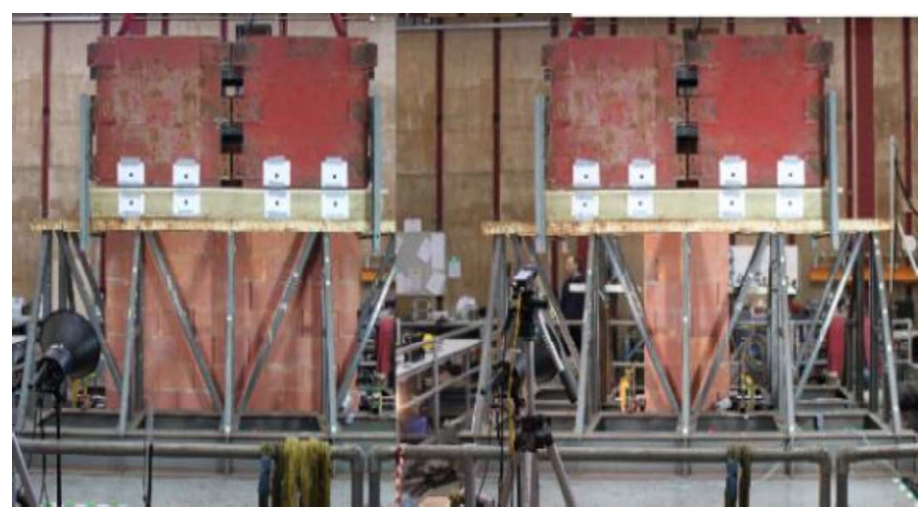

Figure 2: Tested specimens

The present paper is focused on the use of rocking models in view of comparing their predictive capacities with experimental measurements. On the one hand, the results obtained for walls without acoustic elements are assumed to be predictable by the simple model of Hous- 
ner. Several adjustments of the original theory are however needed in order to take into account the upper steel mass lying on the top of the wall. Two main parameters governing the model response are identified, namely the criterion of motion initiation and the restitution coefficient. A two-block model is then used to better describe the tested specimens by properly considering the upper mass, a priori likely to rock with respect to the underlying wall. On the other hand, a new rocking model is derived for the specimens with rubber devices. It aims at modelling two-block structures with viscous and flexible interfaces between the blocks as well as between the bottom block and the foundation. The properties of these interfaces are deduced from experimental data and highly influence the model response.

\section{ROCKING MODELS FOR WALLS WITHOUT RUBBER LAYERS}

A previous contribution of the same authors [31] already tried to reproduce the hereby considered experimental measurements with a model based on the theory developed by Housner. This model was chosen for its simplicity, but some modifications have been performed to fit with the experimental configurations and to consider the additional mass placed on the wall top especially. The results and conclusions of this work are summarized in the first part of this section. The second part is dedicated to the more complex two-block model. This one matches better the experimental reality as the masonry wall and the upper mass are both represented by a specific block. It is however time-consuming in comparison to the simple model (from 2 to 5 times more).

\subsection{Comparison with modified Housner's theory}

Figure 3 illustrates the studied rigid block assuming that the additional steel mass can be considered as a point mass, drawn in red. The mass of the block is assumed to be negligible compared to the top mass. In comparison to the developments of Housner, the consideration of the upper mass involves differences for the parameters $R$ and $\alpha$, representing the radial distance from the centre of rotation $\mathrm{O}$ to the gravity centre and the angle between this distance and the vertical axis respectively.

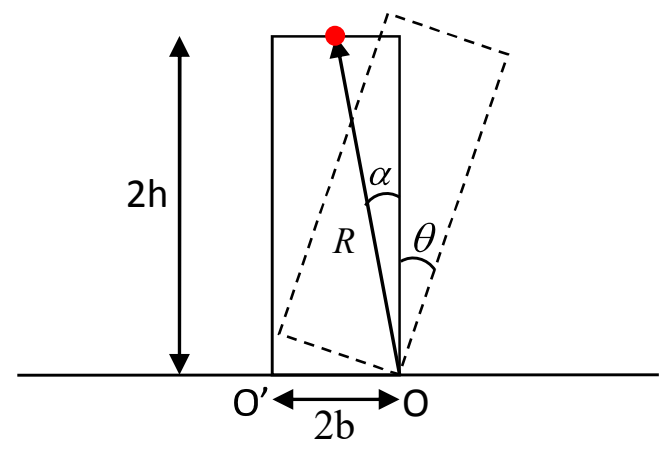

Figure 3: Modified model for simple rigid block

Rocking motion of a single rigid block resting on a rigid foundation and subjected to a horizontal acceleration $\ddot{x}_{g}$ is characterized by its rotation around one of its bottom corners, measured with the positive clockwise variable $\theta$. The following equation describes this motion

$$
I_{O} \ddot{\theta}+M R \ddot{x}_{g} \cos (\alpha \operatorname{sign} \theta-\theta)+M g R \sin (\alpha \operatorname{sign} \theta-\theta)=0
$$

where $\ddot{\theta}\left[\mathrm{s}^{-2}\right]$ is the second time derivative of $\theta[-], I_{O}\left[\mathrm{kgm}^{2}\right]$ is the moment of inertia, $M[\mathrm{~kg}]$ is the mass and $g\left[\mathrm{~ms}^{-2}\right]$ is the gravitational acceleration. 
Rocking is initiated if the overturning moment becomes larger than the restoring one, see eq. (2). The block and the support being rigid, energy dissipation only occurs when the block impacts on the base. This is practically translated by an instantaneous reduction of the angular velocity. Considering no bouncing, the angular momentum around the corner is conserved and allows the quantification of this reduction according to eq. (3).

$$
\begin{gathered}
2 h M \ddot{x}_{g} \geq b M g \\
e=1-2 \frac{M R^{2}}{I_{O}} \sin ^{2} \alpha
\end{gathered}
$$

The equation of motion has been solved using a standard Newmark integration scheme under the assumption of a constant acceleration. The comparisons of numerical predictions with measurements are given in Figure 4 (left) for the short wall and in Figure 4 (right) for the long wall respectively. The results correspond to the last shaking table test with the highest acceleration input, characterized by a PGA of $0.234 \mathrm{~g}$ and $0.688 \mathrm{~g}$ for the short and the long wall respectively.
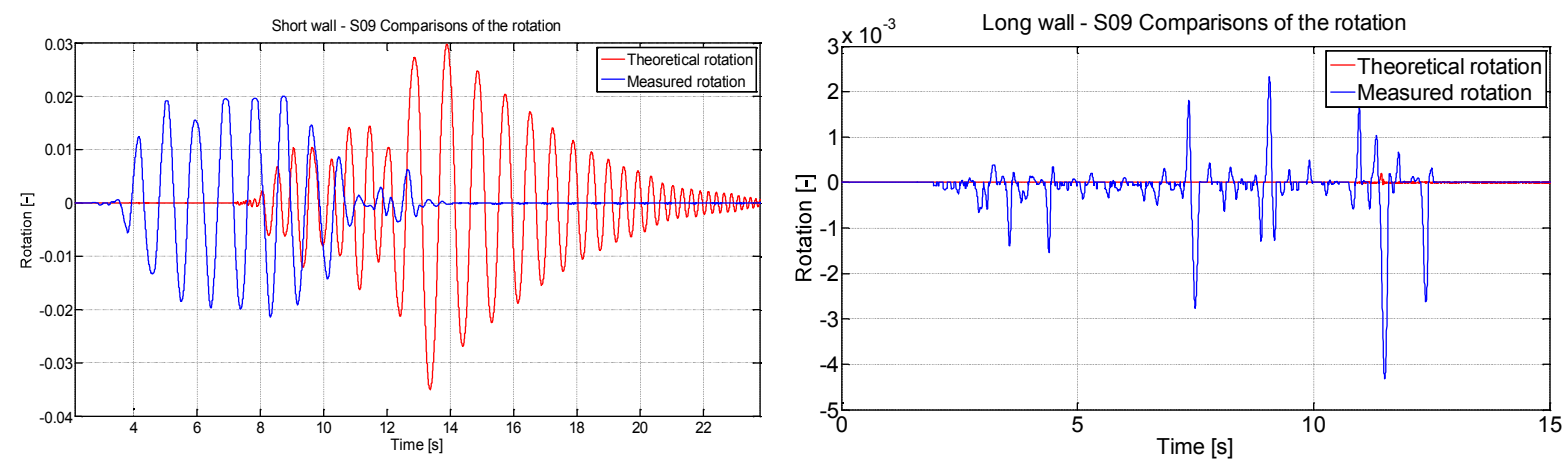

Figure 4: Comparison of the numerical predictions with experimental measurements for the short (left) and long (right) walls without rubber under the assumption of a rigid body

Figure 4 highlights differences between theoretical predictions and experimental measurements. Two issues are identified. First, the initially chosen criterion for defining the initiation of the rocking motion is felt as not appropriate, since the predictions do not capture the moment when rocking starts during the shaking table test on the short wall. This is observed as well for the long wall or for tests with a lower acceleration level for which the numerical simulations do not predict any motion, contrary to the observations. Moreover, the restitution coefficient seems also not to be appropriate. The rotation is indeed getting damped at a slower rate in the model than in reality. Modifications are proposed in [31] and principally consist in discussing the description of the rigid body motion, leading to the choice of a global rotation around an arbitrary point rather than a rotation around the external bottom corners. It results in a change in the parameters $R$ and $\alpha$ as well as in the criterion of motion initiation and restitution coefficient. This latter also integrates a dependence on the square of the aspect ratio and on the ratio of the overall maximum rotation to the one reached during the oscillation before the considered impact. The consequences of these changes on the model predictions are illustrated in Figure 5 for the short wall. 


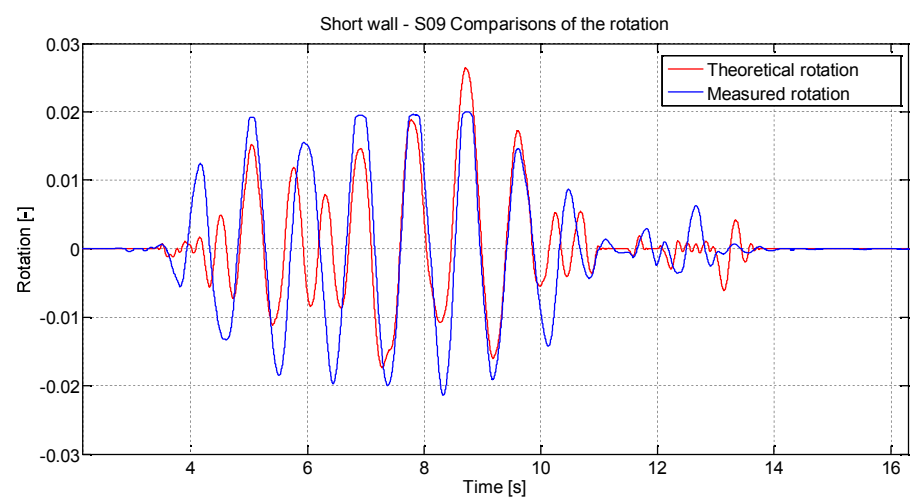

Figure 5: Comparison of the numerical predictions with experimental measurements for the short

The modifications proposed in [31] improve significantly the model predictions. The starting of the rocking motion is better captured and the damping issues at the end of the signal have vanished, albeit some differences remain present. In the case of the long wall, the model is able to predict the occurrence of rotation peak but overestimate their magnitude. Therefore, it can be concluded that such a simple model is not suitable for the present masonry walls. The modelling of the additional mass with a material point located at the block top is identified as a possible reason. A proper consideration of this body should thus enhance the results, which constitutes the topic of the second part of this paper. Another reason for the discrepancies is certainly the need for an educated-guess involved by relaxing the rigid body assumption. Indeed, several possibilities do exist regarding the position of the rotation point. Moreover, the formulation of the restitution coefficient is questionable since the contact between the block and its base is no longer a point.

\subsection{Comparison with a two stacked blocks model}

With regard to the specimen's configuration and to the results presented in the previous section, the two stacked blocks model seems to be more adapted. In this model, the masonry wall and the upper steel mass are indeed each more consistently represented by a block. The equations of motion implemented in the present model are based on the developments of Spanos et al. [26]. Nevertheless, another linearization of these equations is applied and the adopted numerical scheme resorts to an event-driven strategy which principles are detailed in [32] and summarized as follows. The resolution method consists in the integration of the linearized equations of motion corresponding to the current configuration of the system. Once the system configuration changes, the integration is stopped and the event-driven strategy is activated for the handling of the transition. An event localization function is first applied in the perspective of detecting accurately the moment of the transition. The update of the system variables is then operated. The equations of motion corresponding to the new configuration are chosen and the integration restarts. The activation of the event-driven strategy is controlled by "gap" functions defining the conditions to fulfil to change of configurations. In the present case, these "gap" functions are given by the equations translating the transition between configurations with or without impact.

Using the two-block model with the short wall geometry and the acceleration signal as inputs provides the results given in Figure 6. 


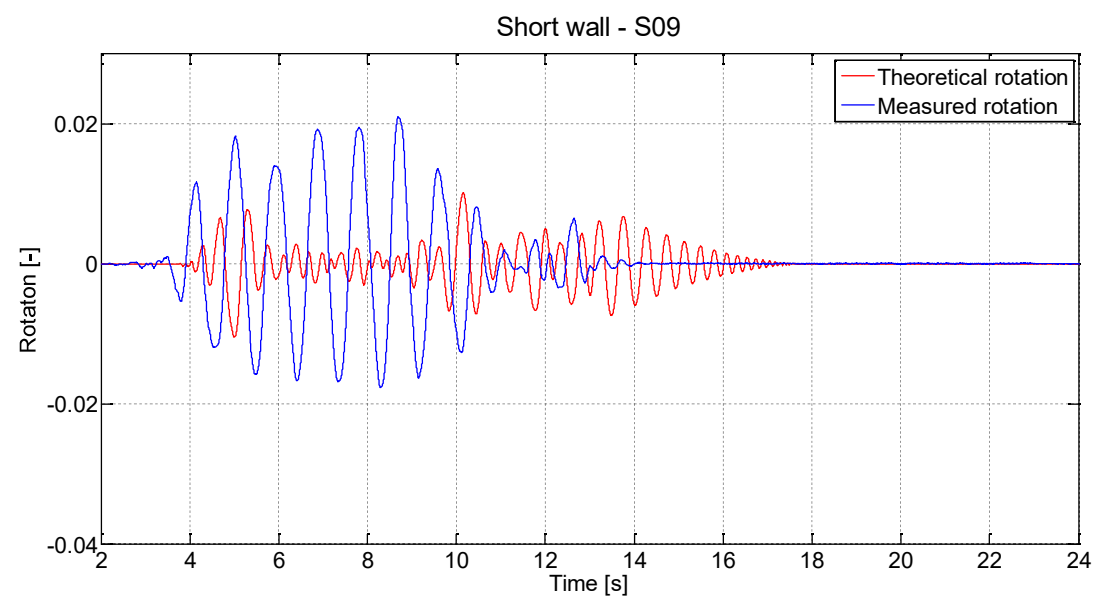

Figure 6: Predictions of the two-stacked-block model for the short wall

As it was observed with the simple model in the previous section (Figure 4), the twostacked-block model cannot reproduce the specimen response. Nonetheless, the prediction of the initiation of the rocking motion is better, as is the energy dissipation. The assumption of a rigid block to model the masonry wall is also questionable. Similar modifications are made to consider a block rotating around another point than its corner, except for the energy dissipation. This leads to the results presented in Figure 7.

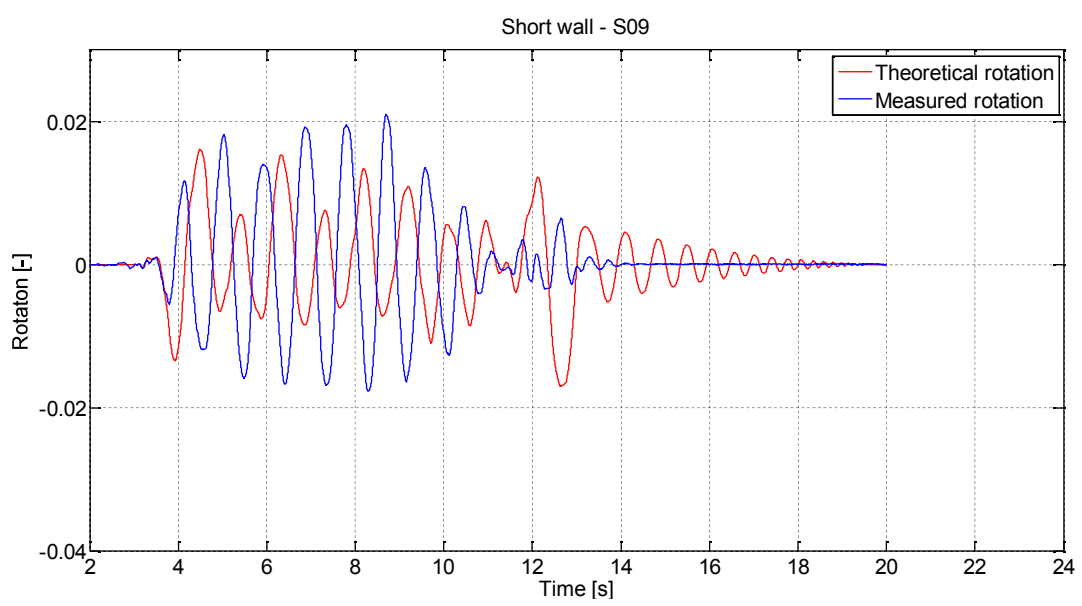

Figure 7: Predictions of the modified two-stacked-block model for the short wall

The initiation of the rocking is now properly captured, but the magnitude of the first theoretical rotation peak is overestimated and occurs too late. This result is possibly due to inadequate account for the energy dissipation. Additional modifications are therefore required. Different attempts have been performed to improve the dissipation of energy at the impacts with a formulation based on the conservation of the angular momentum. These attempts allow a better approximation for the magnitude of the first peak, but the overall response remains remote from the measurements.

As a conclusion, the two stacked blocks model gives better results than the simple model, when based on a similar set of assumptions. Improvements are however required and the assumption of a rigid block for representing the masonry wall seems to be inappropriate. According to the authors, the main reason is the presence of empty vertical joints. Relaxing this assumption to allow rotation around a point to be determined leads to a better prediction of the rocking motion initiation, but makes questionable the quantification of the energy dissipa- 
tion by means of the principle of conservation of the angular momentum. This last point will be the topic of forthcoming investigations.

\section{ROCKING MODEL FOR WALLS WITH RUBBER LAYERS}

Literature review has highlighted a huge interest in the rocking behaviour of single block. Different assumptions have been made on the block, its foundation and the possible behaviours. Besides this, a few research works were focused on the rocking behaviour of two or more stacked blocks. These latter are however limited to rigid blocks standing on a rigid support. This section aims at developing a new rocking model for two-stacked rigid blocks with viscous and flexible interfaces between the support and the bottom block, like blocks standing on a flexible foundation, as well as between the blocks. This model will be then used to compare theoretical predictions with measurements recorded during the shaking table tests on the unreinforced masonry walls including rubber devices.

\subsection{Development of the new rocking model}

The geometry of the modelled blocks is given in Figure 8 (left). The blocks are symmetric and have bases $2 b_{i}$, heights $2 h_{i}$, masses $m_{i}$ and centroid moments of inertia $I_{G i}(i$ is equal to 1 for the base block and 2 for the top block). The centroid of the block is denoted by $G_{i}$. The interfaces have heights $e_{b}$ and $e_{t}$ respectively and are characterized by a stiffness $\left(k_{b}\right.$ or $\left.k_{t}\right)$ and a damping ratio $\left(c_{b}\right.$ or $\left.c_{t}\right)$. Rocking motion of rigid blocks including viscous and flexible interfaces can be described with four variables, namely the rotation of the blocks, $\theta_{1}$ or $\theta_{2}$, and the vertical displacement of the interfaces, $z_{b, 1}$ and $z_{b, 2}$, as denoted in Figure 8 (right). The friction is supposed to be large enough to avoid sliding.

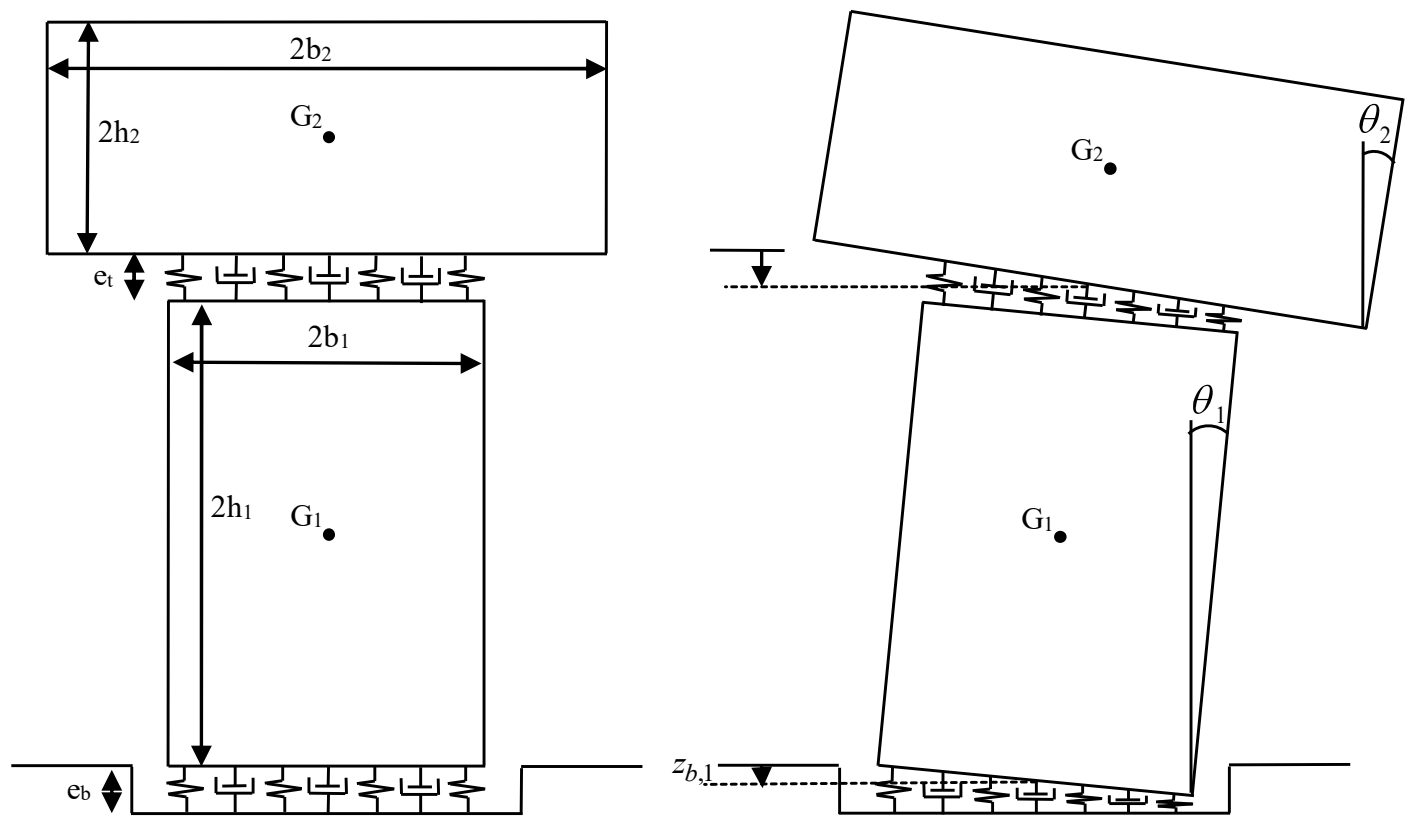

Figure 8: Two-stacked blocks model with viscous and flexible interfaces

It is assumed that the interfaces have no tensile strength. Therefore, uplift occurs when the upward displacement of a part of the base is larger than the deflection due to gravity load. Four different configurations can thus be observed, namely (i) no uplift, (ii) uplift at the base interface, (iii) uplift at the top interface and (iv) uplift at both interfaces. Two parameters $g_{b}$ and $g_{t}$ are defined in order to define the configuration. They are equal to the unit if there is no 
uplift and become zero in the contrary. The conditions to define the value of the parameters are given by (4) and (5).

$$
\begin{aligned}
& g_{b}= \begin{cases}0 & \text { if }\left|z_{b 1}\right|>b_{1} \sin \theta_{1} \\
1 & \text { if }\left|z_{b 1}\right| \leq b_{1} \sin \theta_{1}\end{cases} \\
& g_{t}= \begin{cases}0 & \text { if }\left|z_{b 2}\right|>b_{2} \sin \theta_{2} \\
1 & \text { if }\left|z_{b 2}\right| \leq b_{2} \sin \theta_{2}\end{cases}
\end{aligned}
$$

The equations of motion for the possible configurations are obtained by the Lagrange's method. Details of these equations will be provided in an upcoming contribution. A linearization is then performed and Eq. (6) presents the result in matrix form when the blocks are subjected to seismic horizontal $\ddot{x}_{g}$ and vertical $\dot{z}_{g}$ accelerations.

These linearized equations are implemented in a numerical procedure and is combined with an event-driven strategy for the handling of the transition between configurations. The corresponding "gap" functions are defined by the equations translating the change of value for the two parameters $g_{b}$ and $g_{t}$.

$$
\begin{aligned}
& {\left[\begin{array}{cccc}
I_{G 1}+m_{1} h_{1}^{2}+m_{2}\left(2 h_{1}+e_{t}\right)^{2} & m_{2} h_{2}\left(2 h_{1}+e_{t}\right) & 0 & 0 \\
m_{2} h_{2}\left(2 h_{1}+e_{t}\right) & I_{G 2}+m_{2} h_{2}^{2} & 0 & 0 \\
0 & 0 & m_{1}+m_{2} & m_{2} \\
0 & 0 & m_{2} & m_{2}
\end{array}\right]\left(\begin{array}{c}
\ddot{\theta}_{1} \\
\ddot{\theta}_{2} \\
\ddot{z}_{b, 1} \\
\ddot{z}_{b, 2}
\end{array}\right)} \\
& +\left[\begin{array}{cccc}
-\frac{\left(1+g_{b}\right)}{3} c_{b} b_{1}^{3}+\frac{\left(1+g_{t}\right)}{3} c_{t} b_{1}^{3} & -\frac{\left(1+g_{t}\right)}{3} c_{t} b_{1}^{3} & -\frac{\left(1-g_{b}\right)}{2} c_{b} b_{1}^{2} & \frac{\left(1-g_{t}\right)}{2} c_{t} b_{1}^{2} \\
-\frac{\left(1+g_{t}\right)}{3} c_{t} b_{1}^{3} & \frac{\left(1+g_{t}\right)}{3} c_{t} b_{1}^{3} & 0 & -\frac{\left(1-g_{t}\right)}{2} c_{t} b_{1}^{2} \\
-\frac{\left(1-g_{b}\right)}{2} c_{b} b_{1}^{2} & 0 & \left(1+g_{b}\right) c_{b} b_{1} & 0 \\
\frac{\left(1-g_{t}\right)}{2} c_{t} b_{1}^{2} & -\frac{\left(1-g_{t}\right)}{2} c_{t} b_{1}^{2} & 0 & \left(1+g_{t}\right) c_{t} b_{1}
\end{array}\right]\left(\begin{array}{c}
\dot{\theta}_{1} \\
\dot{\theta}_{2} \\
\dot{z}_{b, 1} \\
\dot{z}_{b, 2}
\end{array}\right) \\
& +\left[\begin{array}{cccc}
-m_{1} g h_{1}-m_{2} g\left(2 h_{1}+e_{t}\right) & \left(1+g_{t}\right) \\
+\frac{\left(1+g_{b}\right)}{3} k_{b} b_{1}^{3}+\frac{\left(1+g_{t}\right)}{3} k_{t} b_{1}^{3} & -\frac{\left(1-g_{b}\right)}{2} k_{b} b_{1}^{2} & \frac{\left(1-g_{t}\right)}{2} k_{t} b_{1}^{2} \\
-\frac{\left(1+g_{t}\right)}{3} k_{t} b_{1}^{3} & -m_{2} g h_{2}+\frac{\left(1+g_{t}\right)}{3} k_{t} b_{1}^{3} & 0 & -\frac{\left(1-g_{t}\right)}{2} k_{t} b_{1}^{2} \\
-\frac{\left(1-g_{b}\right)}{2} k_{b} b_{1}^{2} & 0 & \left(1+g_{b}\right) c_{b} b_{1} & 0 \\
\frac{\left(1-g_{t}\right)}{2} k_{t} b_{1}^{2} & -\frac{\left(1-g_{t}\right)}{2} k_{t} b_{1}^{2} & 0 & \left(1+g_{t}\right) k_{t} b_{1}
\end{array}\right]\left(\begin{array}{c}
\theta_{1} \\
\theta_{2} \\
z_{b, 1} \\
z_{b, 2}
\end{array}\right) \\
& =\left(\begin{array}{c}
-\ddot{x}_{g}\left[m_{1} h_{1}+m_{2}\left(2 h_{1}+e_{t}\right)\right] \\
-\ddot{x}_{g} m_{2} h_{2} \\
-\left(\ddot{z}_{g}+g\right)\left(m_{1}+m_{2}\right) \\
-\left(\ddot{z}_{g}+g\right) m_{2}
\end{array}\right)
\end{aligned}
$$

The energy dissipation in this model is a continuous process. The viscous and flexible interfaces indeed dissipate energy through damping in the dash-pots. 


\subsection{Comparison of the new model with experimental results}

The application of the model requires the calibration of the mechanical properties of the interfaces representing the rubber layers. This is one topic of [33] and is based on seismic tests at a low-to-moderate acceleration level. During these tests, the specimens remained in contact with the support. Deteriorated properties have however been chosen as tests at lower acceleration levels probably damage the rubber devices. Tests at the highest seismic input have been therefore performed on damaged specimens. The interfaces are supposed to behave linearly and elastically. The others model inputs are the blocks geometry and the acceleration signals. The chosen acceleration signals have a PGA of $0.171 \mathrm{~g}$ for the short wall and of $0.457 \mathrm{~g}$ for the long wall. The results in terms of top and bottom rotations are presented in Figure 9 and Figure 10 for the short and the long walls with rubber respectively.
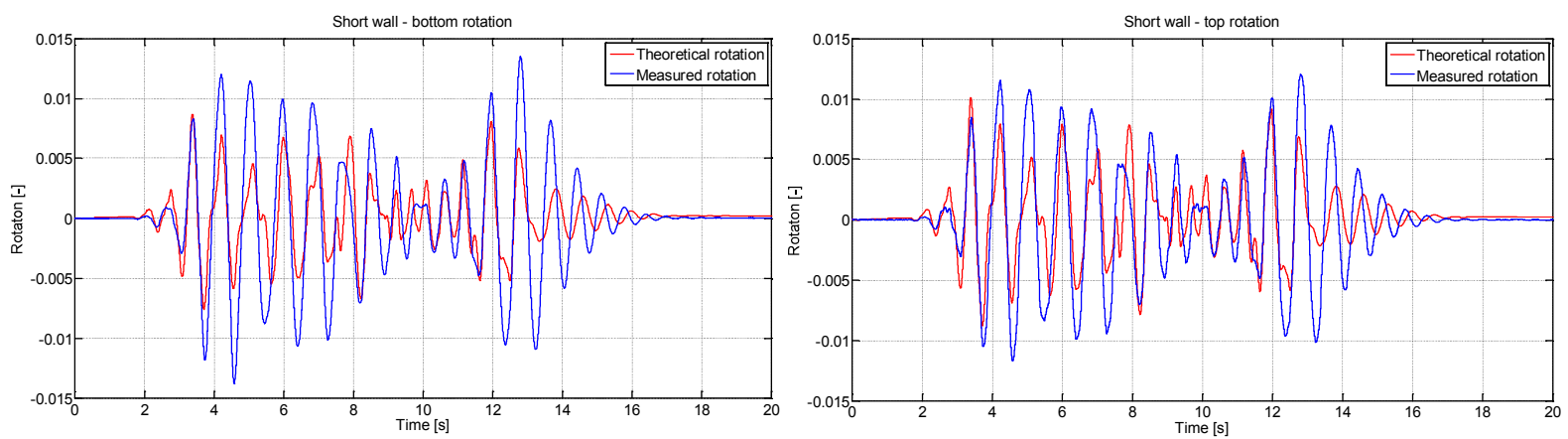

Figure 9: Comparison between model predictions and experimental measurements (short wall)
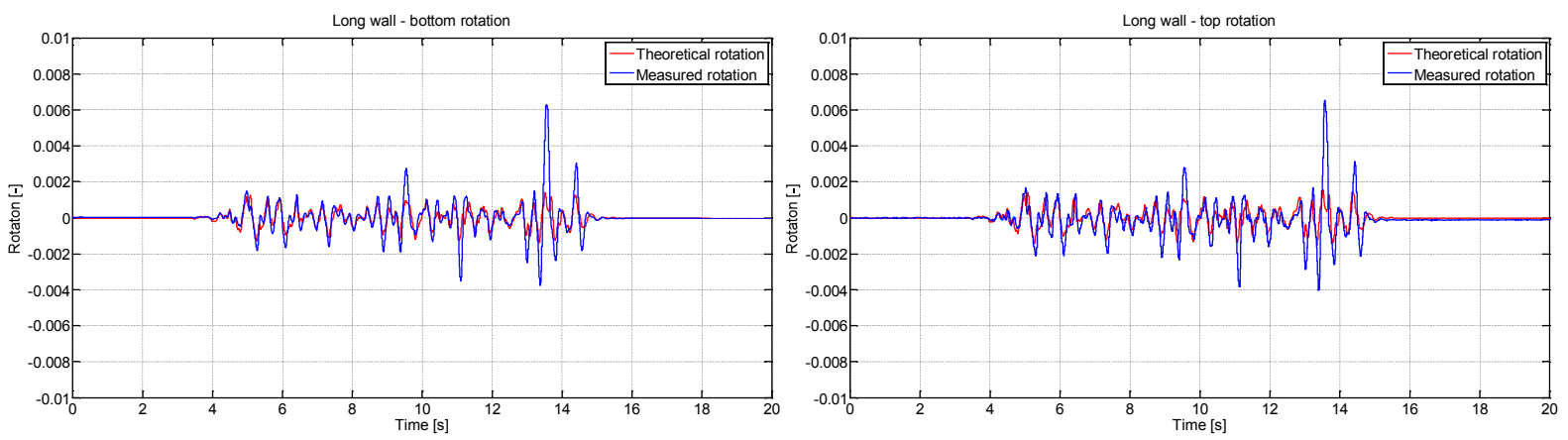

Figure 10: Comparison between model predictions and experimental measurements (long wall)

The mechanical properties used are 4.95 (3.85) MPa for the elastic modulus and 5.44 (1.67) \% for the damping ratio in the case of the short (long) wall. The model predictions are in good agreements with the experimental measurements. The beginning of the rocking motion is well approximated without any further modifications. The assumptions of rigid blocks can indeed be felt here as realistic when referring the rigidity of the block to the one of the joints, which was of course not the case without rubber joints since, in that configuration, the rigidity of the blocks had to be referred to an infinitely rigid foundation. Nevertheless, some differences are observed, especially concerning the peak magnitudes. Consequently, further investigations are required. A larger parameter study is planned to highlight the most relevant properties to be tuned at the level of the interfaces. The possible influence of choosing another type of material law for the rubber layers has also to be studied, these interfaces being the main issue of the model. 


\section{CONCLUSIONS}

This paper deals with the rocking behaviour of masonry structures subjected to seismic loading. The main motivation of this paper is the observation during shake-table tests that a significant rocking motion was experienced by the structures when the tested unreinforced load-bearing clay masonry walls including or not soundproofing rubber layers are shaken at a sufficient acceleration level.

On the one hand, the paper is focused on the rocking behaviour of walls without rubber. A first model is developed, based on the simple theory of Housner and modified to consider the additional mass placed on the walls top. The comparisons of the model predictions with the experimental measurements highlight the need in modelling the additional mass properly and the inconveniency of the assumption of a rigid block for the masonry walls. A second model of two-stacked blocks is therefore implemented with the developments of Spanos et al. This is accompanied with an event-driven strategy for the handling of the transition between configurations. Additional investigations are however still required to allow the masonry walls not to be considered as rigid anymore. The energy dissipation is a main issue since the blocks do not rotate around their corners and, thus, the use of the principle of conservation of the angular momentum is not valid.

On the other hand, a new two-stacked blocks model is developed for the walls with rubber. The main innovation is the consideration of a viscous and flexible interface between the blocks. A good agreement between model prediction and experimental data is found, even if some differences are outlined. In this case, the masonry walls can be assumed as rigid in comparison to the rubber interfaces. The comparison leads to a quite good agreement between theoretical results and experimental measurements. In order to improve the results, further developments are however still in progress, especially regarding the mechanical properties of the interfaces to be used in the model. Details of the new model will be the topic of an upcoming contribution.

\section{ACKNOWLEDGEMENTS}

The research leading to these results has received funding from the European Union Seventh Framework Programme (FP7/2007-2013) under grant agreement $n^{\circ} 227887$, SERIES. C. Mordant also acknowledges the F.R.S - FNRS (Belgian fund for research) for the support of his $\mathrm{PhD}$.

\section{REFERENCES}

[1] G. W. Housner, Behaviour of inverted pendulum structures during earthquakes. Bulletin of the Seismological Society of America, 53(2):403-417, 1963.

[2] C-S. Yim, A.L. Chopra, J. Penzien, Rocking response of rigid blocks to earthquakes, Earthquake engineering and structural dynamics, 8:565-587, 1980.

[3] P. D. Spanos, A-S. Koh, Rocking of Rigid Blocks Due to Harmonic Shaking, Journal of Engineering Mechanics, 110(11):1627-1642, 1984.

[4] J. Zhang, N. Makris, Rocking Response of Free-Standing Blocks Under Cycloidal Pulses, Journal of Engineering Mechanics, 127(5):473-483, 2001.

[5] A. N. Kounadis, On the Overturning Instability of a Rectangular Rigid Block Under Ground Excitation, The Open Mechanics Journal, 4:43-57, 2010. 
[6] F. Prieto, P.B. Lourenço, On the Rocking Behaviour of Rigid Objects, Meccanica, 40:121-133, 2005.

[7] F. Prieto, F. Peña, P.B Lourenço, J. V. Lemos, Numerical Simulation of Rigid Blocks Subjected to Rocking Motion, $5^{\text {th }}$ conference on Structural Analysis of Historic Constructions, New Delhi, India, November, 2006.

[8] I. N. Psycharis, P. C. Jennings, ROcking of Slender Rigid Bodies Allowed to Uplift, Earthquake Engineering and Structural Dynamics, 11:57-76, 1983.

[9] A-S. Koh, P. D. Spanos, J. M. Roesset, Harmonic Rocking of Rigid Block on Flexible Foundation, Journal of Engineering Mechanics, 112(11):1165-1180, 1986.

[10] A. Palmeri, N. Makris, Response analysis of rigid structures rocking on viscoelastic foundation, Earthquake Engineering and Structural Dynamics, 37:1039-1063, 2008.

[11] Q. T. Ma, J. W. Butterworth, Simplified Expressions for Modelling Rigid Rocking Structures on Two-spring Foundations, Annual Technical Conference of NZ Society for Earthquake Engineering, Wellington, New-Zealand, 26-28 March, 2010.

[12] M. A. ElGawady, Q. T. Ma, J. W. Butterworth, J. Ingham, Effects of interface material on the performance of free rocking blocks, Earthquake Engineering and Structural Dynamics, 40:375-392, 2011.

[13] M. F. Vassiliou, N. Makris, Analysis of the rocking response of rigid blocks standing free on a seismically isolated base, Earthquake Engineering and Structural Dynamics, 41:177 -196, 2011.

[14] H. W. Shenton III, N. P. Jones, Base Excitation of Rigid Bodies. I: Formulation, Journal of Engineering Mechanics, 117(11):2286-2306, 1991.

[15] M-Y. Jeong I-Y. Yang, Characterization on the Rocking Vibration of Rigid Blocks under Horizontal Harmonic Excitations, International Journal of Precision Engineering and Manufacturing, 13(2):229-236, 2012.

[16] I. N. Psycharis, Effect of Base Uplift on Dynamic Response of SDOF Structures, Journal of Engineering Mechanics, 117(3):733-754, 1991.

[17] G. Oliveto, I. Caliò, A. Greco, Large displacement behaviour of a structural model with foundation uplift under impulsive and earthquake excitations, Earthquake Engineering and Structural Dynamics, 32:369 -393, 2003.

[18] S. Acikgoz, M. J. DeJong, The interaction of elasticity and rocking in flexible structures allowed to uplift, Earthquake Engineering and Structural Dynamics, 41:2177-2194, 2012 .

[19] R. H. Plaut, W. T. Fielder, L. N. Virgin, Fractal Behaviour of an Asymmetric Rigid Block Overturning Due to Harmonic Motion of a Tilted Foundation, Choas, Solitons \& Fractals, 7(2):177-196, 1996.

[20] R. I. Borosheck, D. Romo, Overturning criteria for non-anchored non-symmetric rigid bodies, 13tg World Conference on Earthquake Engineering, Vancouver, Canada, 1-6 August, 2004.

[21] A. Contento, A. Di Egidio, Investigations into the benefits of base isolation for nonsymmetric rigid blocks, Earthquake Engineering and Structural Dynamics, 38:849 -866, 2009. 
[22] M. J. N. Priestley, R. J. Evison, A. J. Carr, Seismic response of structures free to rock on their foundations, Bulletin of the New Zealand National Society for Earthquake Engineering, 11(3):141-150, 1978.

[23] N. Makris, D. Konstantinidis, The rocking spectrum and the limitations of practical design methodologies, Earthquake Engineering and Structural Dynamics, 32:265-289, 2003.

[24] T. E. Kelly, Tentative seismic design guidelines for rocking structures, Bulletin of the New Zealand National Society for Earthquake Engineering, 42(4):239-274, 2009.

[25] I. N. Psycharis, Dynamic behaviour of rocking two-block assemblies, Earthquake Engineering and Structural Dynamics, 19:555-575, 1990.

[26] P. D. Spanos, P. C. Roussis, N. P. A. Politis, Dynamic analysis of stacked rigid blocks, Soil Dynamics and Earthquake Engineering, 21:559-578, 2001.

[27] A. N. Kounadis, G. J. Papadopoulos, D. M. Costovos, Overturning instability of a tworigid block system under ground excitation, Journal of Applied Mathematics and Mechanics, 92(7):536-557, 2012.

[28] M. J. DeJong, E. G. Dimitakopoulos, Dynamically equivalent rocking structures, Earthquake Engineering and Structural Dynamics, 43:1543-1563, 2014.

[29] F. Peña, F. Prieto, P. B. Lourenço, A. C. Costa, Experimental dynamic behavior of free standing multi-block structures under seismic loadings, Journal of Earthquake Engineering, 12:953-979, 2008.

[30] C. Mordant, M. Dietz, H. Degée, Seismic behaviour of thin-bed layered unreinforced clay masonry shear walls including soundproofing elements, In: Alper Ilki and Michael N. Fardis (eds), Proc SERIES Workshop. Geotechnical, Geological and Earthquake Engineering series (chapter 6).

[31] C. Mordant, M. Dietz, H. Degée, Shaking table tests on unreinforced load-bearing masonry walls - comparison with simple rocking models (Paper $\mathrm{n}^{\circ} 1238$ ), In: Proc. 4th ECCOMAS Thematic Conference on Computational Methods in Structural Dynamics and Earthquake Engineering (COMPDYN 2013), eds. M. Papadrakakis, V. Papadopoulos \& V. Plevris, Kos Island, Greece, 12-14 June 2013

[32] A. Depouhon, E. Detournay, V. Denoël, Event-driven integration of linear structural dynamics models under unilateral elastic constraints, Computer Method in Applied Mechanics and Engineering, 276:312-340, 2014.

[33] C. Mordant, C. Taylor, M. Dietz, L. Vasseur, H. Degée, Shaking table tests on unreinforced load-bearing masonry structures, $9^{\text {th }}$ International Masonry Conference, Guimarães, Portugal, 7-9 July, 2014. 